\title{
Numerical Simulation of Unsteady Incompressible Viscous Flows in Generalized Coordinate Systems
}

Moshe Rosenfeld

Dochan Kwak, Ames Research Center, Moffett Field, California

July 1988

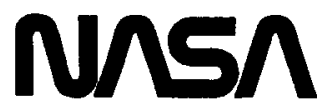

National Aeronautics and Space Administration 


\title{
NUMERICAL SIMULATION OF UNSTEADY INCOMPRESSIBLE VISCOUS FLOWS IN GENERALIZED COORDINATE SYSTEMS
}

\author{
Moshe Rosenfeld* and Dochan Kwak ${ }^{\dagger}$ \\ NASA Ames Research Center, Moffett Field, CA 94035
}

\section{Summary}

Several numerical solutions of the three-dimensional unsteady incompressible Navier-Stokes equations in generalized coordinate systems are presented in this work. The governing equations are discretized by finite volumes with special care to the accurate approximation of the geometric quantities. The unknowns are the pressure and the volume fluxes over the computational cell faces. This formulation results in a robust fractional-step solution method for solving the discrete equations. Although the method is formulated for the three-dimensional case, only two-dimensional unsteady results are given in this work. Results are presented for a lid-driven two-dimensional cavity flow at Reynolds number of $10^{4}$, and for the flow over a circular cylinder with vortex shedding for several Reynolds numbers in the range $100<R e<1000$.

\section{Introduction}

Numerous fluid flow phenomena are essentially time-dependent. Until recently, the numerical simulation of unsteady flow fields over complicated configurations was very difficult because of limited computer resources both in terms of processor speed and storage. The new large supercomputers are powerful enough to make the unsteady flow simulation possible, provided an efficient and accurate solution procedure can be devised.

This work presents a numerical simulation method for viscous time-dependent incompressible flow fields in arbitrary geometries. Two principal approaches have been used for the solution of the incompressible Navier-Stokes equations in primitive formulation [1]. One method is based on the fractional-step solution procedure while the other uses the artificial-compressibility concept with dual-time stepping for restoring the time-accuracy of the solution. The present study uses a fractional-step solution method. An efficient and robust solution procedure has been developed and several test cases have been computed to validate the method. The details of the numerical procedure and some of the validation cases are presented in Refs. [2] and [3]. Therefore, only a brief description of the method is given here and a few solutions are included.

*NRC Research Associate

tResearch Scientist 


\section{Formulation}

Navier-Stokes equations govem the flow of isothermal, constant-density, incompressible fluids. For a control volume $V$ with surface $S$ the mass conservation equation is

$$
\oint_{S} d \mathbf{S} \cdot \mathbf{u}=0
$$

and the momentum conservation equation is

$$
\frac{d}{d t} \int_{V} \mathbf{u} d V=\oint_{S} d \mathbf{S} \cdot \bar{T},
$$

where $\mathbf{u}$ is the velocity vector, $t$ is the time, $d \mathbf{S}$ is a surface-area element, and $d V$ is a volume-element. For Newtonian fluids the tensor $\bar{T}$ is given by

$$
\bar{T}=-\mathbf{u u}-P \bar{I}+\nu\left(\nabla \mathbf{u}+(\nabla \mathbf{u})^{T}\right),
$$

where $P$ is the pressure, $\nu$ is the effective kinematic viscosity, $\bar{I}$ is the identity tensor, $\nabla \mathbf{u}$ is the gradient of $\mathbf{u}$, and $(\cdot)^{T}$ is the transpose operator.

\section{Discretization and Numerical Solution}

Equations (1-3) are directly discretized over finite volumes to yield a fully conservative second-order scheme. Finitevolume discretization is preferred since it usually results in more accurate and stable solutions for generalized coordinate systems, especially for meshes with heavily clustered points and large curvature. Special care is given to satisfy the "geometrical conservation laws" [2] to minimize the errors resulting from the spatial discretization.

The numerical problems associated with the absence of a pressure time-derivative in the mass conservation equation are handled by a fractional-step procedure. In each time step, the momentum equations are solved for an approximate velocity field which does not satisfy the continuity equation. In the second stage, the velocity and pressure fields are corrected such that the mass conservation equation is satisfied. This step leads to a Poisson equation with Neumann-type boundary conditions which may exhibit very poor convergence properties, especially in generalized coordinate systems. The CPU time consumed by the Poisson solver may be as high as $80 \%$ of the total computational time even in a Cartesian case [4]. In the present work an attempt is made to minimize these difficulties.

This attempt has led to the choice of the pressure (defined at the center of the computational cells) and the volume fluxes across each computational cell face as the dependent variables instead of the familiar Cartesian components of the velocity. (The volume fluxes are equivalent to the scaled contravariant velocity components in a staggered grid.) This choice ensures the satisfaction of the discrete mass conservation equation to round-off errors in any coordinate system and has favorable effect on the convergence properties of the Poisson solver (see details in Refs. [2] and [3]).

A consistent Poisson solver is obtained by deriving the Laplacian operator from the discrete equivalent of the operator $\nabla \cdot \nabla$ (where $\nabla \cdot$ and $\nabla$ are the divergence and the gradient operators, respectively), rather than discretizing the Laplacian operator directly. A novel and efficient ZEBRA scheme with four-color ordering is devised for the efficient solution of the nonorthogonal Poisson equation on vector computers. The Poisson solver could be converged to any specified small error in all the cases solved so far. 


\section{Results}

Various test cases were solved to validate the present solution method. The three-dimensional flow in a cubic cavity as well as the symmetric flow over a circular cylinder at $R e=40$ and at $R e=200$ with vortex shedding are described in Ref. [2]. The present paper reports additional cases to assess further the capabilities of the method. All the cases were solved using the three-dimensional code. Two-dimensional solutions were obtained by specifying along the third direction two intervals and periodic boundary conditions.

\subsection{Lid-driven Cavity, $\operatorname{Re}=10^{4}$}

In this case the time-dependent flow in a lid-driven square cavity was solved for a Reynolds number of $10^{4}$. A Cartesian nonuniform grid of $81 \times 81 \times 3$ mesh points was used with clustering near the walls. The minimal interval is $0.28 \cdot 10^{-2}$ and the maximal interval at the center is $0.23 \cdot 10^{-1}$. The upper lid started moving impulsively at $T=0$ and the time-accurate flow field was computed until the nondimensional time $T=120$ was reached. Previously, steady solutions of this case were reported in the literature, see for example Ghia et al. [5]. However, these solutions were not obtained by a time-accurate method. For $R e=10^{3}$, the steady solution obtained by the present solution procedure compares favorably with other computations (see Ref. [3]).

For the case $R e=10^{4}$ the evolution of the main vortex and of the secondary vortices at the comers of the cavity exhibit complicated patterns that are repeated almost periodically without approaching a steady state. Figure 1 shows the instantaneous streamlines for $102>T>92$ which corresponds approximately to one "period." During this time interval, the main vortex rotates around a fictitious center and the flow field at the lower two comers goes through a complicated series of states which include the generation of secondary and tertiary vortices and their merging with one another. Recently, Bruneau and Jouron [6] have found that for this case the solution has not converged to a steady state if a fine grid is used. However, no experimental results are available for comparison at this time.

\subsection{Symmetrical Flow over a Circular Cylinder}

The symmetrical flow field over a circular cylinder at $R e=40$ was solved employing a nonorthogonal grid with $45 \times 73 \times 3$ mesh points in the radial, circumferential, and axial directions, respectively. Figure 2 compares the time-evolution of the separation length with other numerical and experimental results. Good agreement is obtained, especially for $T<6$. Slight disagreement is found for $T>6$ due to the "wall effect," which is the result of the finite distance of the outer boundary and the Dirichlet-type boundary conditions given in the present solution. This wall effect was studied earlier by varying the outer boundary distance (see Ref. [2]).

\subsection{Vortex Shedding from a Circular Cylinder}

This case considers the shedding of vortices behind a circular cylinder at Reynolds numbers 100-1000. A cylindrical grid of $81 \times 84 \times 3$ mesh points was employed with points clustered near the cylinder and in the wake region. The vortex shedding was triggered by rotating the cylinder for a short period of time in both clockwise and counterclockwise directions. Figure 3 shows the time evolution of the lift coefficient (dashed line) and the drag coefficient (full line) for several Reynolds numbers. In each case, the onset of the vortex-shedding and the periodic flowfield can be observed after a relatively short transient flow. Figure 4 compares the Strouhal number with other experimental and numerical results given in Refs. [9]-[11]. The present solution yields a somewhat high Strouhal number for all the range of the Reynolds numbers. This behavior was also found by Gresho et al. [10]. Figure 5 shows the instantaneous streamlines for Reynolds numbers $100,200,550$, and 1000 at the instant which corresponds to the maximal lift coefficient. 


\section{Concluding Remarks}

A time-accurate method for the solution of the incompressible Navier-Stokes equations is developed based on a fractional-step method. The dependent variables are the pressure and the volume fluxes across the computational cells. The goveming equations are discretized by the finite-volume method with special care to satisfy the geometric conservation laws. The method is second-order-accurate both spatially and temporarily.

The computed results compare favorably with other numerical and experimental studies. In the present paper some of the numerical results for the flow in a lid-driven square cavity and over a circular cylinder are presented.

\section{References}

[1] Kwak, D., Chang, J. L. C., Rogers, S. E. and Rosenfeld, M., "Three-Dimensional Incompressible NavierStokes Computations of Intemal Flows," Proc. of the 12th IMACS World Congress on Scientific Computation, Paris, France, July 18-22, 1988 (also NASA TM 100076, 1988).

[2] Rosenfeld, M., Kwak, D. and Vinokur, M., "A Solution Method for the Unsteady and Incompressible NavierStokes Equations in Generalized Coordinate Systems," AIAA-88-0718, 1988.

[3] Rosenfeld, M., Kwak, D. and Vinokur, M., "Development of an Accurate Solution Method for the Unsteady Incompressible Navier-Stokes Equations in Generalized Coordinate Systems," NASA TM, in process, 1988

[4] Van Doormaal, J. P. and Raithby, G. D., "Enhancements of the SIMPLE Method for Predicting Incompressible Fluid Flows," Num. Heat Transfer, 7, 1984, pp. 147-163.

[5] Ghia, U., Ghia, K. N. and Shin, C. T., "High-Re Solutions for Incompressible Flow Using the Navier-Stokes Equations and a Multigrid Method," J. Comp. Phys., 48, 1982, pp. 387-411.

[6] Bruneau, C. H. and Jouron, C., "An Efficient Scheme for Solving Steady Incompressible Navier-Stokes Equations," Université de PARIS-SUD MATHÉMATIQUES Prépublications 88-22, 1988.

[7] Collins, W. M. and Dennis, S. C. R., "Flow Past an Impulsively Started Circular Cylinder," J. Fluid Mech., 60, 1973, pp. 105-127.

[8] Coutanceau, M. and Bouard, R., "Experimental Determination of the Main Features of the Viscous Flow in the Wake of a Circular Cylinder in Uniform Translation, Part 2. Unsteady Flow," J. Fluid Mech., 79, 1977, pp. 257-272.

[9] Braza, M., Chassaing, P. and Ha Minh, H., "Numerical Study and Physical Analysis of the Pressure and Velocity Fields in the Near Wake of a Circular Cylinder," J. Fluid Mech., 165, 1986, pp. 79-130.

[10] Gresho, P. M., Chan, S. T., Lee, L. and Upson, C. D., "A Modified Finite Element Method for Solving the Time-Dependent, Incompressible Navier-Stokes Equations. Part 2: Applications," Int. J. Num. Meth. Fluids, 4, 1984, pp. 619-640.

[11] Roshko, A., "On the Development of Turbulent 'Vakes from Vortex Streets," NACA TN 2913, 1953. 


$$
\begin{aligned}
& \text { ORIGINAL PAGE IS } \\
& \text { OF POOR QUALTY }
\end{aligned} \quad T=98
$$

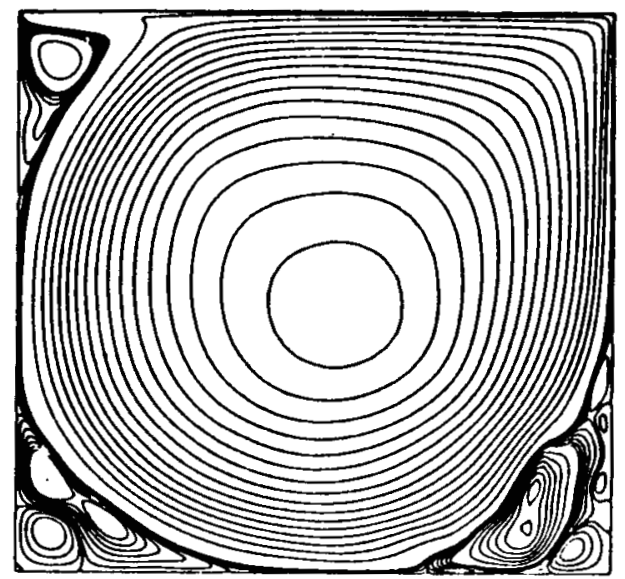

$$
T=94
$$

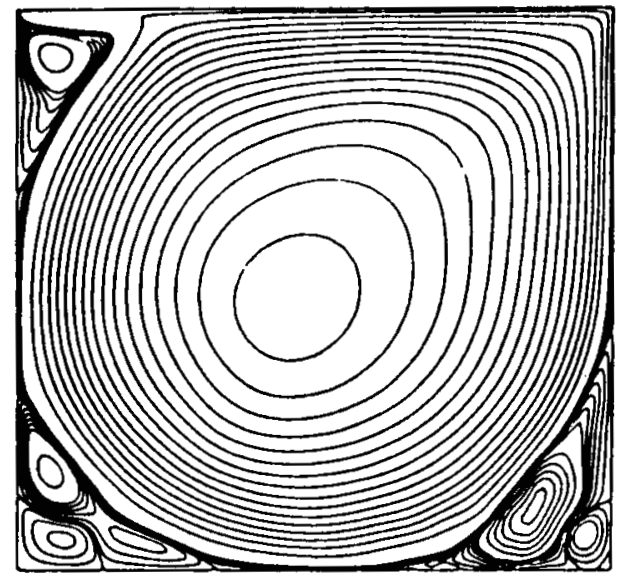

$$
T=96
$$

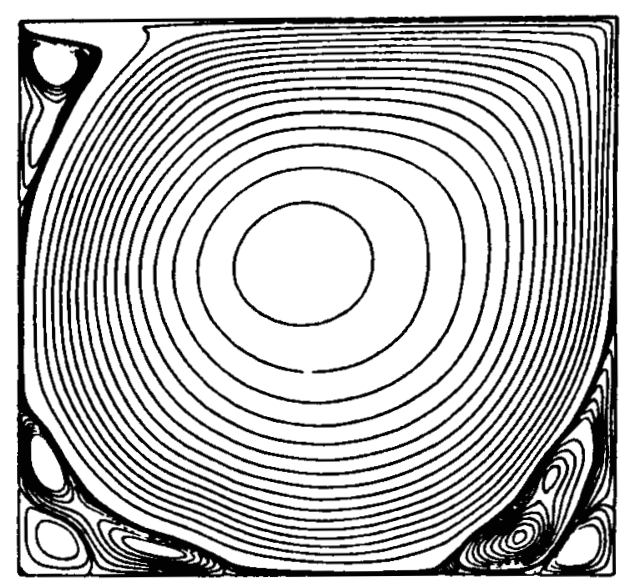

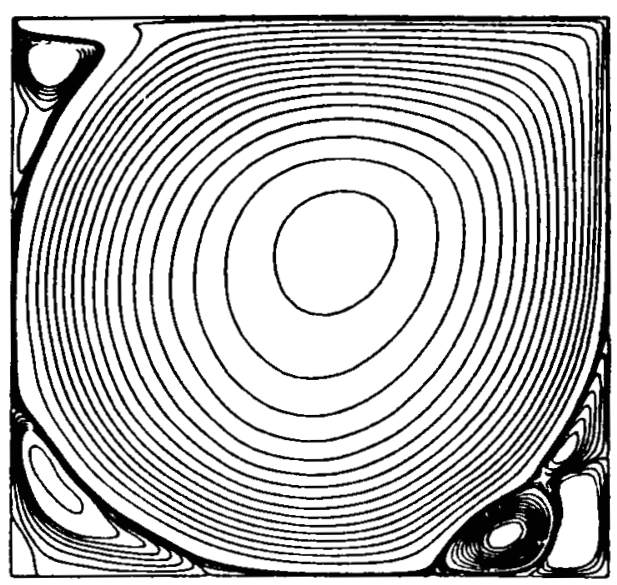

$$
T=100
$$

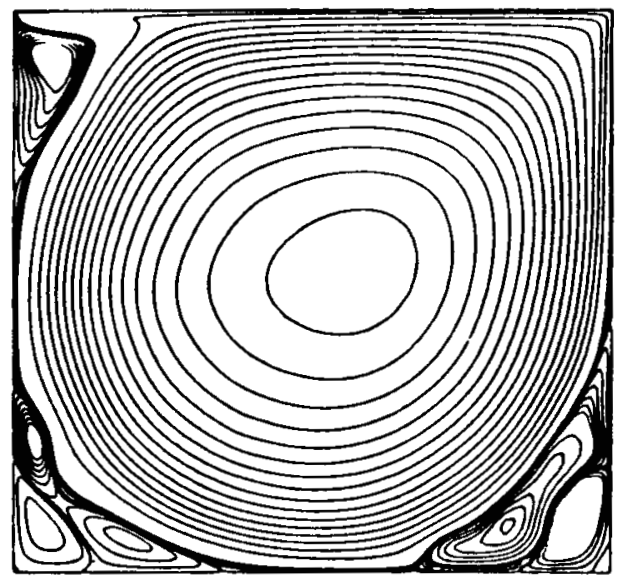

$$
T=102
$$

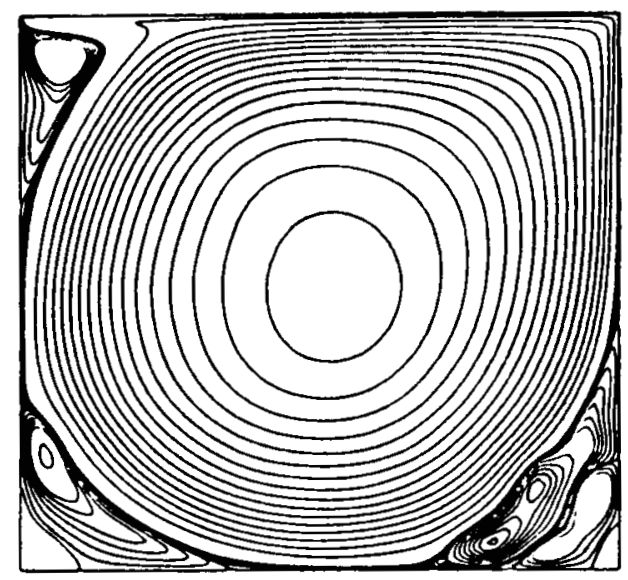

Figure 1: Instantaneous streamlines, $R e=10^{4}$ 


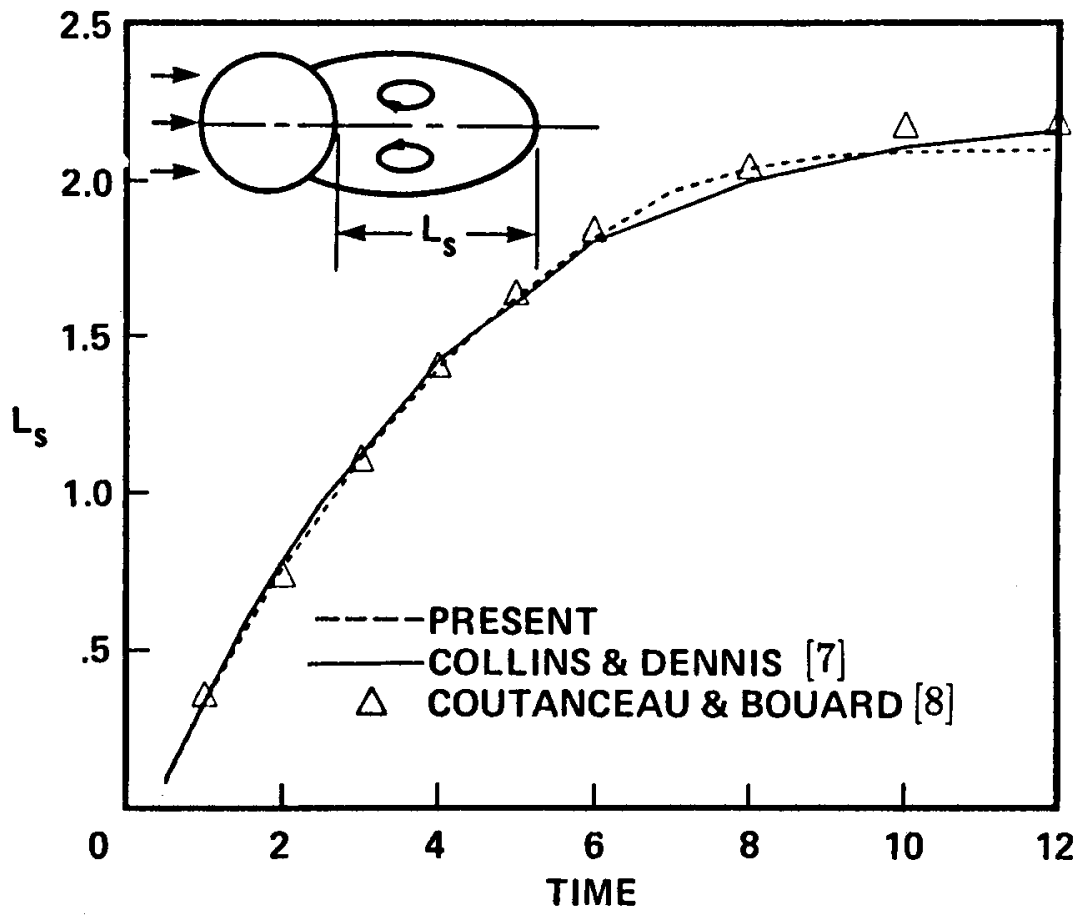

Figure 2: Time-evolution of the separation-length $(R e=40)$ 

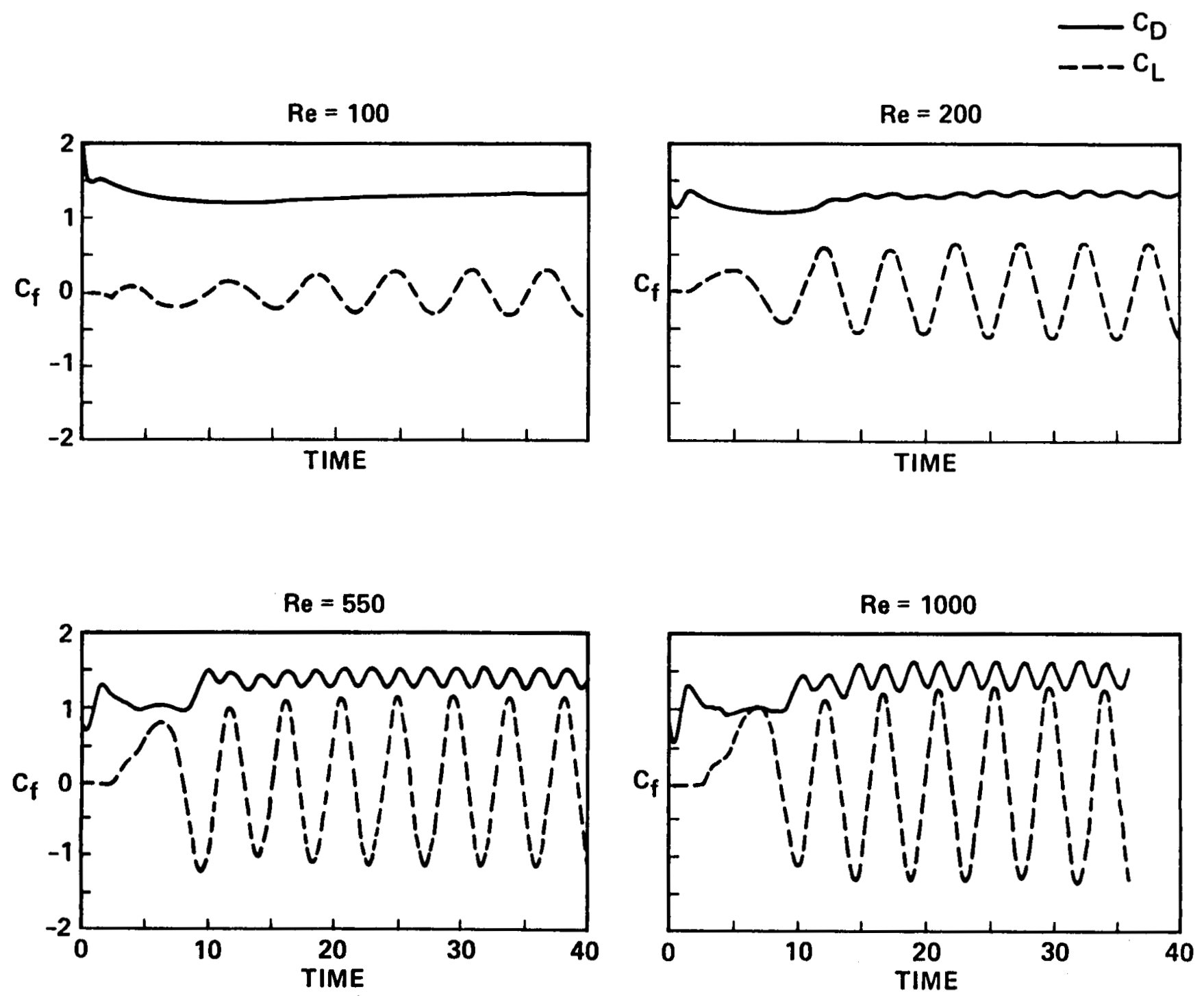

Figure 3: Time-evolution of the force coefficients 


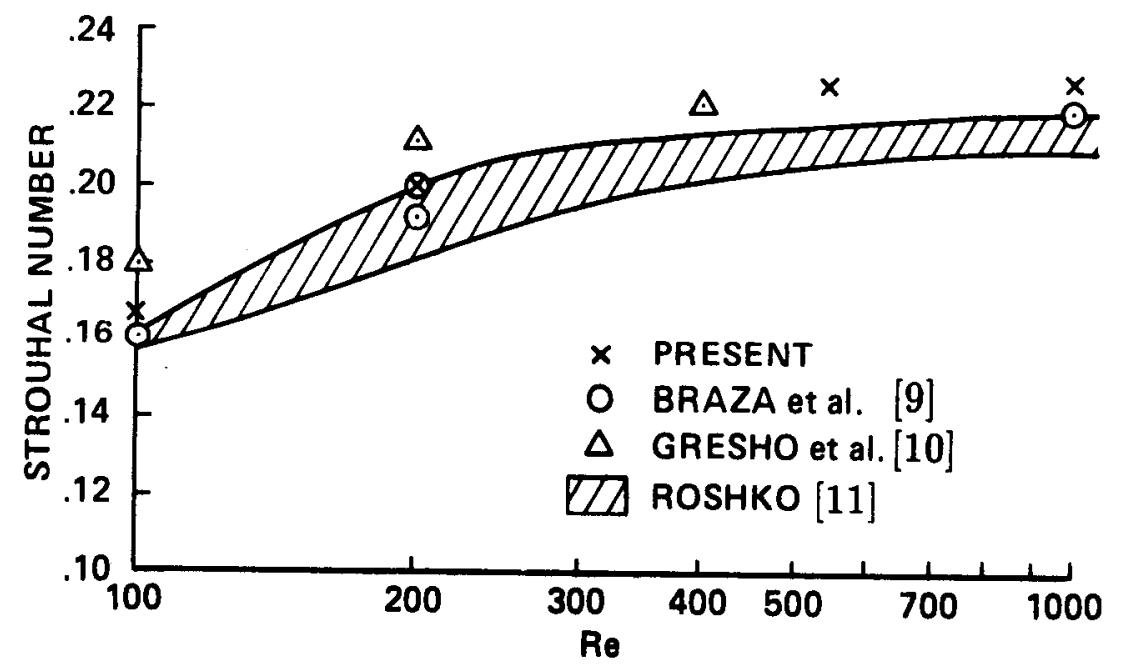

Figure 4: Dependence of the Strouhal number on the Reynolds number (circular cylinder) 


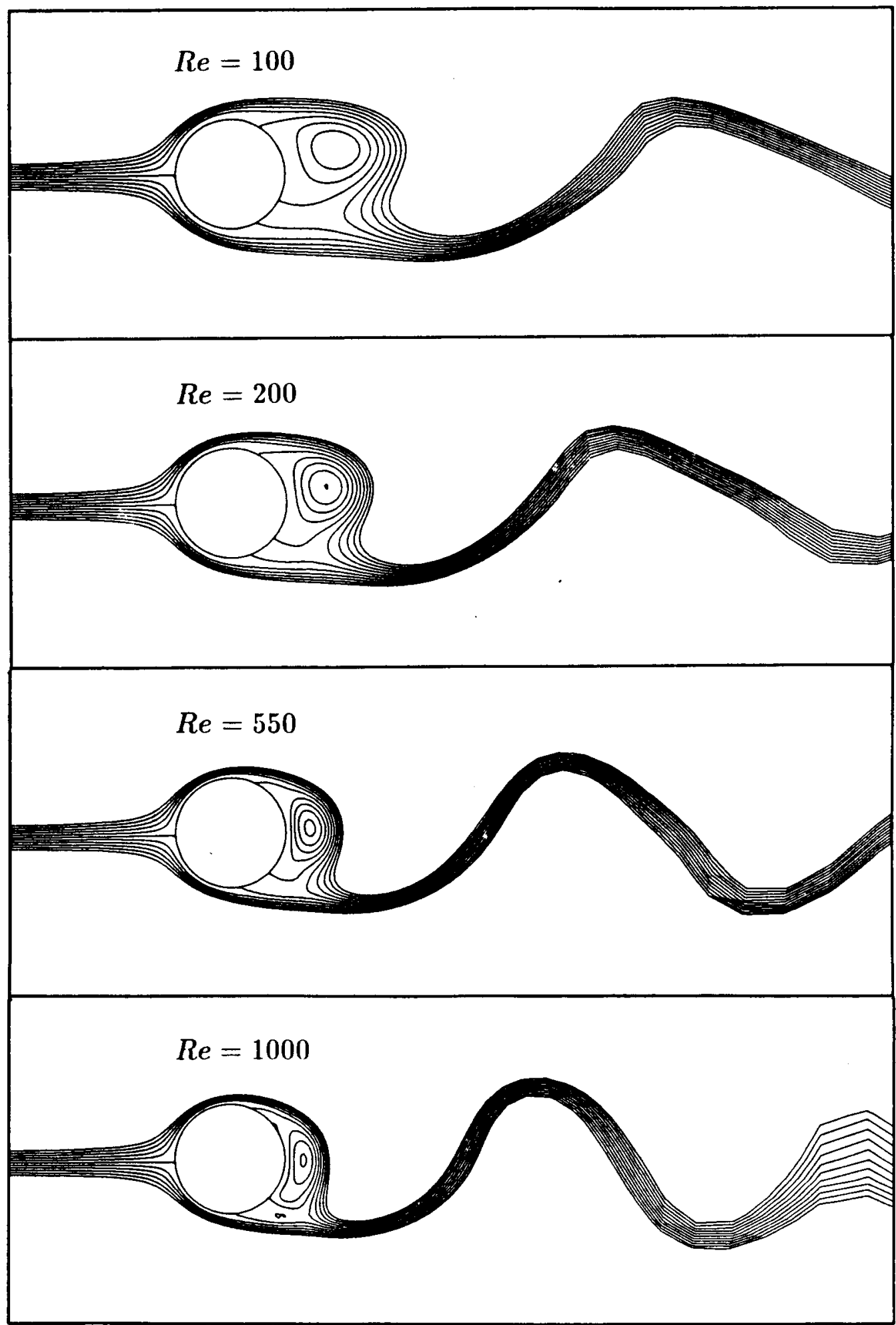

Figure 5: Effect of the Reynolds number on the instantaneous streamlines 


\begin{tabular}{|c|c|c|c|}
\hline \multicolumn{4}{|c|}{ Report Documentation Page } \\
\hline $\begin{array}{l}\text { 1. Report No. } \\
\text { NASA TM-101016 }\end{array}$ & 2. Government Accession No. & \multicolumn{2}{|c|}{ 3. Recipient's Catalog No. } \\
\hline \multirow{2}{*}{\multicolumn{2}{|c|}{$\begin{array}{l}\text { 4. Title and Subtitle } \\
\text { Numerical Simulation of Unsteady Incompressible Viscous } \\
\text { Flows in Generalized Coordinate Systems }\end{array}$}} & \multicolumn{2}{|l|}{$\begin{array}{l}\text { 5. Report Date } \\
\text { July } 1988\end{array}$} \\
\hline & & \multicolumn{2}{|c|}{ 6. Performing Organization Code } \\
\hline \multicolumn{2}{|l|}{ 7. Author(s) } & \multicolumn{2}{|c|}{$\begin{array}{l}\text { 8. Performing Organization Report No. } \\
\text { A-88254 }\end{array}$} \\
\hline \multicolumn{2}{|c|}{ Moshe Rosenfeld and Dochan Kwak } & \multicolumn{2}{|l|}{ 10. Work Unit No. } \\
\hline \multicolumn{2}{|c|}{ 9. Performing Organization Name and Address } & \multicolumn{2}{|l|}{$505-60$} \\
\hline \multicolumn{2}{|l|}{$\begin{array}{l}\text { Ames Research Center } \\
\text { Moffett Field, CA } 94035\end{array}$} & 11. Contract or $\mathrm{Gr}$ & \\
\hline \multirow{2}{*}{\multicolumn{2}{|c|}{$\begin{array}{l}\text { 12. Sponsoring Agency Name and Address } \\
\text { National Aeronautics and Space Administration } \\
\text { Washington, D.C. } 20546-0001\end{array}$}} & \multicolumn{2}{|c|}{$\begin{array}{l}\text { 13. Type of Report and Period Covered } \\
\text { Technical Memorandum }\end{array}$} \\
\hline & & \multicolumn{2}{|c|}{ 14. Sponsoring Agency Code } \\
\hline \multicolumn{4}{|c|}{$\begin{array}{l}\text { Point of Contact: Dochan Kwak, Ames Research Center, MS 258-1, Moffett Field, CA } 94035 \\
\text { (415) 694-6743 or FTS 464-6743 }\end{array}$} \\
\hline \multicolumn{4}{|c|}{$\begin{array}{l}\text { 16. Abstract } \\
\text { Several numerical solutions of the three-dimensional unsteady incompressible Navier-Stokes equations in gener- } \\
\text { alized coordinate systems are presented in this work. The governing equations are discretized by finite volumes } \\
\text { with special care to the accurate approximation of the geometric quantities. The unknowns are the pressure and } \\
\text { the volume fluxes over the computational cell faces. This formulation results in a robust fractional-step solution } \\
\text { method for solving discrete equations. Although the method is formulated for the three-dimensional case, only two- } \\
\text { dimensional unsteady results are given in this work. Results are presented for a lid-driven two-dimensional cavity } \\
\text { flow at Reynolds number of } 10^{4} \text {, and for the flow over a circular cylinder with vortex shedding for several Reynolds } \\
\text { numbers in the range } 100<R e<1000 \text {. }\end{array}$} \\
\hline 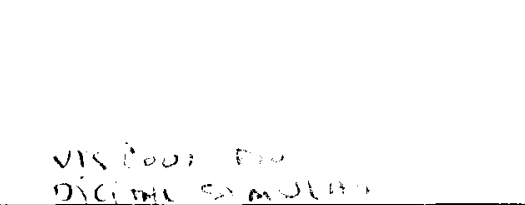 & & $\therefore$ & \\
\hline $\begin{array}{l}\text { 17. Key Words (Suggested by Author(s)) } \\
\text { Incompressible flow } \\
\text { Unsteady flow } \\
\text { Navier-Stokes equations } \\
\text { Finite volume }\end{array}$ & 18. Dist & Unlimited & \\
\hline $\begin{array}{l}\text { 19. Security Classif. (of this report) } \\
\text { Unclassified }\end{array}$ & $\begin{array}{l}\text { 20. Security Classif. (of this pagel } \\
\text { Unclassified }\end{array}$ & $\begin{array}{l}\text { 21. No. of pages } \\
11\end{array}$ & $\begin{array}{r}\text { 22. Price } \\
\text { A02 }\end{array}$ \\
\hline
\end{tabular}

